\title{
Microsymposium
}

MS85.001

\section{Structure of PhnZ reveals a novel oxygenase mechanism for $C-P$ bond cleavage}

L. van Staalduinen ${ }^{1}$, F. McSorley ${ }^{2}$, D. Zechel ${ }^{2}$, Z. Jia $^{1}$

${ }^{1}$ Queen's University, Department of Biomedical and Molecular Sciences, Kingston, Canada, ${ }^{2}$ Queen's University, Department of Chemistry, Kingston,

Canada

Inorganic phosphate is an essential component of many biological molecules and processes including cellular signaling, generation of metabolic energy, DNA and RNA, and membrane phospholipids. Organophosphonates, which contain a highly stable carbonphosphorus bond, are widely used as herbicidal, chelation, anti-scale, and medicinal agents. PhnY and PhnZ consist of a new oxidative catabolic pathway that is employed by marine bacteria to use 2-aminoethylphosphonic acid as a source of inorganic phosphate. PhnZ is notable for catalyzing the oxidative cleavage of a carbon-phosphorus bond using Fe(II) and dioxygen (see figure), despite belonging to a large family of hydrolytic enzymes, the HD-phosphohydrolase superfamily. We have determined structures of PhnZ in complex with its substrate, (R)-2-amino-1-hydroxyethylphosphonate. The structure reveals PhnZ to have an active site containing two Fe ions bound by 4 histidines and 2 aspartates (see figure) that is strikingly similar to the carbon-carbon bond cleaving enzyme, myo-inositoloxygenase. Site-directed mutagenesis and kinetic analysis with substrate analogues revealed the roles of key active site residues. A 5th histidine that is conserved in the PhnZ subfamily specifically interacts with the substrate 1-hydroxyl. The structure also revealed that PhnZ possesses a unique induced-fit mechanism whereby an active-site aspartate specifically recognizes the 2-amino group of the substrate and toggles the release of an aromatic residue from the active site, thereby creating space for molecular oxygen bind to the second Fe ion. Structural comparisons of PhnZ reveal an evolutionary connection between Fe(II)-dependent hydrolysis of phosphate esters and oxidative carbon-phosphorus or carbon-carbon bond cleavage, thus uniting the diverse chemistries that are found in the HD superfamily.

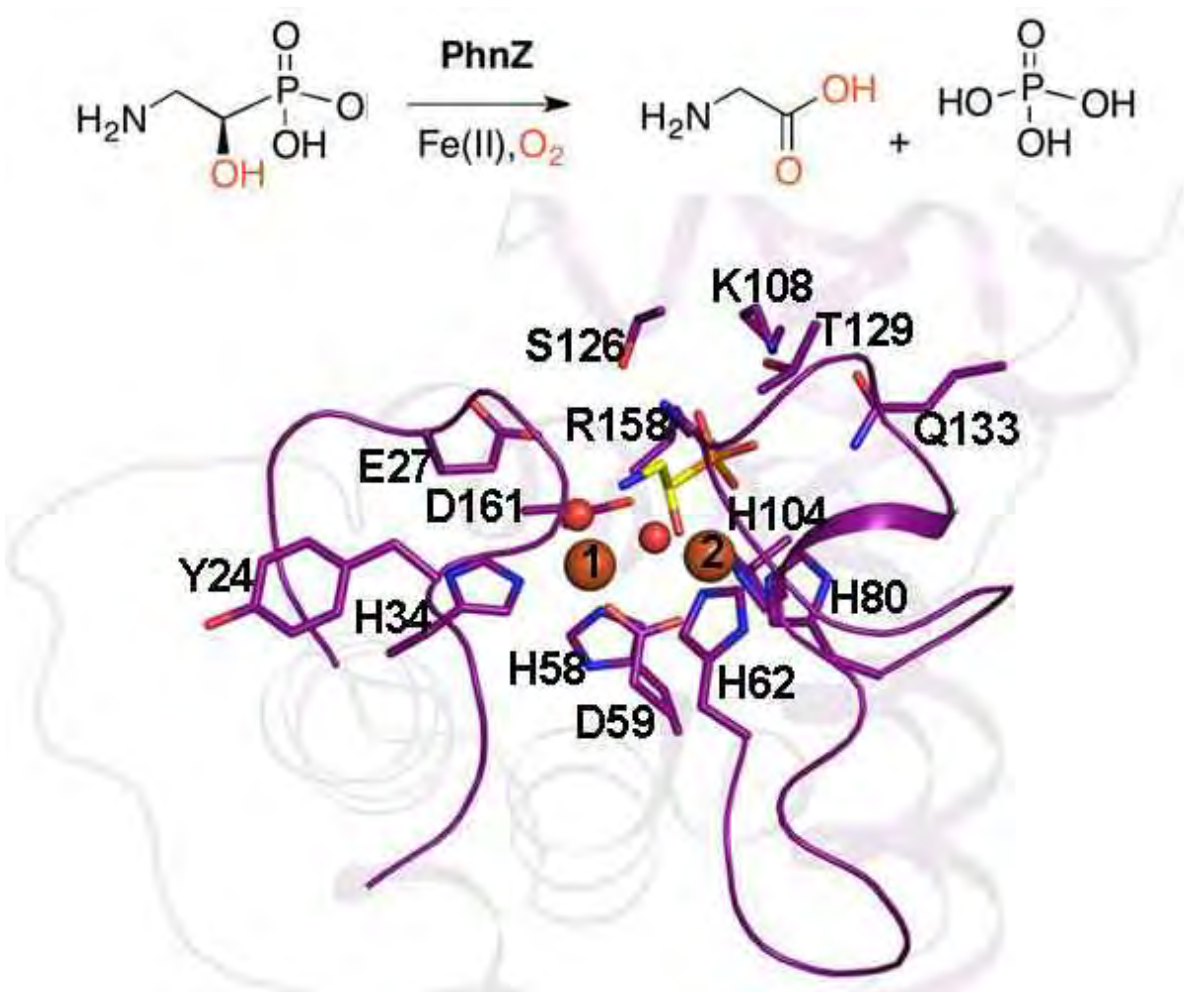

Keywords: carbon-phosphorus bond cleavage, enzyme-substrate complex, mixed valence di-iron oxygenase 